\title{
Gravitini zero modes riding on top of multiple black holes
}

\author{
Palash Dubey and Chethan N. Gowdigere \\ School of Physical Sciences, National Institute of Science Education and Research, Jatni-752050, India \\ and Homi Bhabha National Institute, Training School Complex, Anushakti Nagar, Mumbai 400094, India
}

(Received 16 October 2020; accepted 13 May 2021; published 10 June 2021)

\begin{abstract}
We study gravitini zero modes in four dimensional $\mathcal{N}=2$ pure supergravity theory. The gravitini zero modes we study are solutions to the Rarita-Schwinger equations of motion in the background of the purely bosonic Majumdar-Papapetrou background. We start with a very generic ansatz for the gravitini that involves 32 ansatz functions and reduce the Rarita-Schwinger equations to a set of linear coupled partial differential equations on $\mathbf{R}^{3}$ : the kind familiar from electromagnetism with divergences, curls, etc. We first show how the gravitini zero modes due to broken supersymmetries arise in this setup and how they solve the equations on $\mathbf{R}^{3}$. Then we go on and obtain other solutions to these equations: the "extra" fermion zero modes, parametrized by four integration constants (one of the integration constants corresponds to the gravitini zero modes due to broken supersymmetries). A careful analysis of pure gauge solutions reveals that we have four independent extra zero modes.
\end{abstract}

DOI: 10.1103/PhysRevD.103.126011

\section{INTRODUCTION}

We study fermion zero modes riding on top of purely bosonic supersymmetric solutions in four dimensional supergravity. The supergravity theory we study is the $\mathcal{N}=2$ pure supergravity [1], which is a theory of a graviton, a graviphoton and a two gravitinis. The purely bosonic solution we focus on is the multicenter MajumdarPapatetrou [2,3] black hole solution, which is known to be supersymmetric [4-6] preserving half and breaking the other half of the supersymmetries of the action.

An important properly of purely bosonic supersymmetric solutions is that they admit fermion zero modes which are classical solutions to the fermionic equations of motion, which in this case are the Rarita-Schwinger equations, in the background of the bosonic solution. It is straightforward to generate some of these fermion zero modes, the ones corresponding to the super symmetries broken by the bosonic solution (the goldstinos). The fermion zero modes corresponding to broken supersymmetries are obtained by performing a supersymmetry transformation on the bosonic solution using a supersymmetry parameter, that at asymptotic infinity is orthogonal to the Killing spinors of the bosonic solution; these supersymmetry parameters are referred to as anti-Killing spinors. The resulting configuration of fields is such that the bosonic fields are unchanged

Published by the American Physical Society under the terms of the Creative Commons Attribution 4.0 International license. Further distribution of this work must maintain attribution to the author(s) and the published article's title, journal citation, and DOI. Funded by SCOAP ${ }^{3}$. but the fermionic fields are now nonzero. This nonzero fermionic field thus obtained is guaranteed to solve the fermionic equations of motion in the bosonic background. We work out the explicit form of these fermion zero modes due to broken supersymmetry here in this paper, reproducing [7] the work done in a series of papers by Aichelburg et al. [8-13].

In this paper, we go on and provide a computational setup for and explicitly obtain fermion zero modes in the background of the purely bosonic Majumdar-Papapetrou supersymmetric solution. We come up with an ansatz for the fermions and directly solve the fermion equations of motion. We first find the fermion zero mode due to broken supersymmetries as one of the solutions to the fermion equations of motion; then we obtain new, hitherto unknown, solutions. These "extra fermion zero modes" are the main result of this paper.

It is rare to find studies which directly solve the equations of motion for the fermion fields in a supergravity theory, which is what we do in this paper. One reason for this rarity is that it is cumbersome and becomes technically hard very soon. In this paper, in which we deal with a comparatively easier supergravity theory, the $\mathcal{N}=2$ pure supergravity theory with only one fermion field, our ansatz involves a total of 32 ansatz functions and when plugged into the Rarita-Schwinger equation of motion results in a total of 32 coupled linear partial differential equations. We can reduce these equations into a manageable form; we group the ansatz functions into scalar and vector and ranktwo tensor fields on a $\mathbf{R}^{3}$ and the equations pertain to the gradients and curls and divergences of these fields and are in the form that is familiar from ordinary electromagnetism. 
We then manage to solve these equations and obtain explicit solutions with four integration constants; see (56). Some of the solutions (pertaining to one of the integration constants, $C$ in (56) are not new viz. the fermion zero modes corresponding to broken super symmetries. While it is important to recognize that these solutions can be obtained without actually solving the fermion equations of motion and just by performing a supersymmetry transformation on the bosonic solution, it is even more important to recognize that the other solutions [pertaining to the remaining three integration constants, $D_{1}, D_{2}, D_{3}$ in (56)] are genuinely new results of our study and could not have been obtained in any other way except by going through the long analysis that will be laid out in this paper. When one examines the main result of this paper viz. the solution in (56), it may seem like is it is a mere generalization of previously known solutions (setting the $D$ 's to zero gives the fermion zero modes corresponding to broken supersymmetries), we should reiterate that it is anything but that.

Most of the papers on this subject [7-17] were written decades ago. The motivation for those papers was the study of supersymmetric solitons in supergravity theories and more particularly finding the supersymmetric theory that describes the dynamics of these solitons. For the Majumdar-Papapetrou black hole solitons, the theory was found in [17]. A more recent motivation for this work comes from the microscopic theory for the entropy of multiple supersymmetric black holes. In certain cases, considerations coming from microscopic formulas, indicate that the macroscopic bosonic supersymmetric black hole solution should admit "extra" fermion zero mode solutions; the word extra refers to the fact that these solutions are above and beyond the fermion zero modes due to broken supersymmetries. Extra fermion zero modes are expected in multicenter Denef-Bates [18] solutions which are solutions that generically preserve one quarter of the supersymmetries of four dimensional $\mathcal{N}=4$ supergravity. Some references for extra fermion zero modes are [19-21] [22]. Both the bosonic solutions viz. the Denef-Bates solution and the supergravity theory viz. the $\mathcal{N}=4$ theory are very involved. Here in this paper, we study this problem of finding fermion zero modes in the simplest possible setting. That is, for the supersymmetric MajumdarPapapetrou multi-black holes in four dimensional $\mathcal{N}=2$ pure supergravity. We hope [23] the setup and the results of this paper go some way in getting to the above mentioned goal.

This paper is organized as follows. In the following Sec. II we first review relevant details of the supergravity, the supersymmetry transformations and the MajumdarPapapetrou multicenter black hole solutions. We construct the $\hat{\mathcal{D}}$ operator for the Majumdar-Papapetrou solution which is the operator that enters into our computations in three different contexts: (i) in the equation to determine the Killing spinors, (ii) in the formulas to generate the fermion zero modes due to broken supersymmetries and (iii) in the Rarita-Schwinger equations of motion that the fermion zero modes solve. In Sec. II, we first compute the Killing spinors and then compute the anti-Killing spinors and with those for supersymmetry parameters generate the fermion zero modes due to broken supersymmetries. In Sec. III, first we motivate the ansatz for the fermions, which turns out to have a total of 32 real functions and then proceed to compute the consequence of the fermion equations of motion on these ansatz functions. It turns out that the 32 functions form two decoupled sectors of 16 functions each; the functions in each sector are coupled among themselves in a set of coupled first order linear partial differential equations. We give the equations for both the sectors in this section and we also discuss the $\gamma$ tracelessness gauge condition that we work with through out the paper. Then, in Sec. IV, we solve the equations of motion in one of the decoupled sectors, the one that includes the ansatz functions that are turned on in the fermion zero mode due to broken supersymmetry. Before solving, we show that the fermion zero mode due to broken supersymmetries indeed solves the equation of motion. Then we present the analysis of the equations that leads to the extra fermion zero modes. In this section (IV), we also compute the supercharges of the new solutions and study pure gauge solutions and present a counting of the independent zero modes. In the final section (V) we conclude with a discussion and directions for future work. An appendix gathers all the equations of motion in each decoupled sector.

\section{GRAVITINI ZERO MODES DUE TO BROKEN SUPERSYMMETRIES}

In this section, we first review the supergravity theory and the supersymmetric solutions and subsequently compute the Killing spinors and the fermion zero modes due to broken supersymmetry.

\section{A. Four dimensional $\mathcal{N}=2$ pure supergravity}

The fundamental fields of four dimensional $\mathcal{N}=2$ pure supergravity are (i) the gravitational field written in terms of the vierbien fields $e^{a}=e_{\mu}^{a} d x^{\mu}$, (ii) the electromagnetic potential one-form $A=A_{\mu} d x^{\mu}$ and (iii) two Majorana spinor-valued one-forms $\psi^{j}, j=1,2$ combined to a complex Dirac field $\psi=\psi^{1}+i \psi^{2}=\psi_{\mu} d x^{\mu}$. All fields are oneforms: the bosonic fields are ordinary one-forms, i.e., taking values in space-time functions and the fermionic field is a one-form taking values in Dirac spinors. The indices corresponding to one-form components are suppressed.

The two supersymmetry transformation parameters $\epsilon^{j}, j=1,2$ are Majorana spinor fields, combined into a complex Dirac spinor field $\epsilon=\epsilon^{1}+i \epsilon^{2}$. The supersymmetry transformations of the theory are 


$$
\begin{aligned}
\delta e^{a} & =-\frac{i k}{2}\left[\bar{\epsilon} \gamma^{a} \psi-\bar{\psi} \gamma^{a} \epsilon\right], \quad \delta A=\frac{i}{2}[\bar{\epsilon} \psi-\bar{\psi} \epsilon], \\
\delta \psi & =\frac{1}{k} \hat{\mathcal{D}} \epsilon .
\end{aligned}
$$

where

$$
\hat{\mathcal{D}}=d+\frac{1}{2} \omega^{a b} \sigma_{a b}-\frac{k}{2} \hat{F}^{a b} \sigma_{a b} \gamma
$$

with

$$
\hat{F}=F-\frac{i k}{2} \bar{\psi} \wedge \psi, \quad \gamma \equiv \gamma_{a} e^{a} .
$$

$\omega^{a b}$ is the spin-connection one-form and $F$ is the electromagnetic field strength two-form and $\gamma$ is a Clifford-algebra valued one-form built out of the vierbien one-forms. We work with the conventions of $[9,14,15,17]$. The space-time signature is mostly negative and $k^{2}=4 \pi G$ and we use a Weyl representation for the $\gamma$-matrices [16].

The action of four dimensional $\mathcal{N}=2$ supergravity, which can be found in [1], which we will not write here, is invariant under the supersymmetry transformations (1). Among the equations of motion, we will need explicitly only the linearized fermionic equations of motion:

$$
\gamma \wedge \hat{\mathcal{D}} \wedge \psi=0
$$

The $\hat{\mathcal{D}}$ that is in (4) is the linearized version of the one in (2). This same linearized version is also relevant for the action of supersymmetry on a purely bosonic configuration and is given explicitly below. In the rest of the paper $\hat{\mathcal{D}}$ will denote this linearized version.

$$
\hat{\mathcal{D}}=d+\frac{1}{2} \omega^{a b} \sigma_{a b}-\frac{k}{2} F^{a b} \sigma_{a b} \gamma \equiv d+\omega F
$$

$\hat{\mathcal{D}}$ is an operator taking values in the Clifford-algebra: the first term $d$ has no $\gamma$ matrices, the second term $\frac{1}{2} \omega^{a b} \sigma_{a b}$ has two $\gamma$ matrices and the third term $-\frac{k}{2} F^{a b} \sigma_{a b} \gamma$ has one or three $\gamma$ matrices. The second and third terms of $\hat{\mathcal{D}}$, together denoted by $\omega F$, is a Clifford-algebra valued one-form. The action of $\hat{\mathcal{D}}$ is on a spinor (which can be thought of as a spinor valued zero form) to produce a spinor valued oneform: $d$ acts on spinor valued zero-forms and produces spinor valued one-forms while $\omega F$ being Clifford-algebra valued acts on a spinor to produce a spinor and since it is also a one-form it produces a spinor-valued one-form in the end.

\section{B. Majumdar-Papapetrou multicenter black holes}

The Majumdar-Papapetrou (MP) solution [2,3] is a purely bosonic solution to four dimensional $\mathcal{N}=2$ pure supergravity describing multiple extremal static black holes. The nonvanishing fields on the MP solution are $e^{0}=\frac{d t}{V}, \quad e^{1}=V d x, \quad e^{2}=V d y, \quad e^{3}=V d z, \quad A=-\frac{d t}{k V}$.

For the following choice of the function $V$,

$$
V(x, y, z)=1+\sum_{J=1}^{n} \frac{G M_{J}}{\left|\vec{x}-\vec{x}_{J}\right|}, \quad \vec{x}_{J} \neq \vec{x}_{K}, \quad J \neq K
$$

this bosonic configuration (6) describes $n$ static extremal charged black holes with mass parameters $M_{J}$ and electric charges $k M_{J}$. Without loss of generality, we restrict ourselves to the case of positive electric charges and zero magnetic charges. The sign of all electric charges may be reversed by changing the sign of $A$, while magnetic charges can be generated by a duality transformation. The spatial part of the metric is conformal to a $\mathbf{R}^{3}$ with coordinates $x, y, z$.

\section{The $\hat{\mathcal{D}}$ operator for the Majumdar-Papapetrou solution}

There is a $\hat{\mathcal{D}}$ operator (5) for every bosonic background. We first construct the $\hat{\mathcal{D}}$ operator for the MP solution. This is relevant to compute the Killing spinors of the MP solution as well as to compute the fermion zero modes in the MP solution.

$$
\hat{\mathcal{D}}=d+\omega F
$$

where $\omega F$ is the following Clifford-algebra valued oneform:

$$
\begin{array}{r}
e^{0}\left[\frac{\partial_{x} V}{2 V^{2}} \gamma_{1}\left(\mathbf{1}-\gamma_{0}\right)+\frac{\partial_{y} V}{2 V^{2}} \gamma_{2}\left(\mathbf{1}-\gamma_{0}\right)+\frac{\partial_{z} V}{2 V^{2}} \gamma_{3}\left(\mathbf{1}-\gamma_{0}\right)\right] \\
+e^{1}\left[\frac{\partial_{x} V}{2 V^{2}} \gamma_{0}-\frac{\partial_{y} V}{2 V^{2}} \gamma_{1} \gamma_{2}\left(\mathbf{1}-\gamma_{0}\right)+\frac{\partial_{z} V}{2 V^{2}} \gamma_{3} \gamma_{1}\left(\mathbf{1}-\gamma_{0}\right)\right] \\
+e^{2}\left[\frac{\partial_{y} V}{2 V^{2}} \gamma_{0}-\frac{\partial_{z} V}{2 V^{2}} \gamma_{2} \gamma_{3}\left(\mathbf{1}-\gamma_{0}\right)+\frac{\partial_{x} V}{2 V^{2}} \gamma_{1} \gamma_{2}\left(\mathbf{1}-\gamma_{0}\right)\right] \\
+e^{3}\left[\frac{\partial_{z} V}{2 V^{2}} \gamma_{0}-\frac{\partial_{x} V}{2 V^{2}} \gamma_{3} \gamma_{1}\left(\mathbf{1}-\gamma_{0}\right)+\frac{\partial_{y} V}{2 V^{2}} \gamma_{2} \gamma_{3}\left(\mathbf{1}-\gamma_{0}\right)\right]
\end{array}
$$

Killing spinor fields, $\epsilon_{K S}$ are those, when used as supersymmetry parameters in a supersymmetry transformation on a configuration of fields, leaves the configuration invariant; such an invariant configuration of fields is termed supersymmetric. The MP solution is a supersymmetric solution to $4 d \mathcal{N}=2$ supergravity; this was shown in [4-6]. For a purely bosonic configuration of fields, such as the MP solution, the Killing spinors solve the following vanishing-fermion-variation equation

$$
\hat{\mathcal{D}} \epsilon_{K S}=0
$$


the bosonic-variation-equations are trivially satisfied due to the vanishing of the fermion fields in the purely bosonic configuration. From Eq. (8), we have

$$
\begin{aligned}
0= & e^{0}\left[V \partial_{t} \epsilon_{K S}+\frac{\partial_{x} V}{2 V^{2}} \gamma_{1}\left(\mathbf{1}-\gamma_{0}\right) \epsilon_{K S}+\frac{\partial_{y} V}{2 V^{2}} \gamma_{2}\left(\mathbf{1}-\gamma_{0}\right) \epsilon_{K S}+\frac{\partial_{z} V}{2 V^{2}} \gamma_{3}\left(\mathbf{1}-\gamma_{0}\right) \epsilon_{K S}\right] \\
& +e^{1}\left[\frac{\partial_{x} \epsilon_{K S}}{V}+\frac{\partial_{x} V}{2 V^{2}} \gamma_{0} \epsilon_{K S}-\frac{\partial_{y} V}{2 V^{2}} \gamma_{1} \gamma_{2}\left(\mathbf{1}-\gamma_{0}\right) \epsilon_{K S}+\frac{\partial_{z} V}{2 V^{2}} \gamma_{3} \gamma_{1}\left(\mathbf{1}-\gamma_{0}\right) \epsilon_{K S}\right] \\
& +e^{2}\left[\frac{\partial_{y} \epsilon_{K S}}{V}+\frac{\partial_{y} V}{2 V^{2}} \gamma_{0} \epsilon_{K S}-\frac{\partial_{z} V}{2 V^{2}} \gamma_{2} \gamma_{3}\left(\mathbf{1}-\gamma_{0}\right) \epsilon_{K S}+\frac{\partial_{x} V}{2 V^{2}} \gamma_{1} \gamma_{2}\left(\mathbf{1}-\gamma_{0}\right) \epsilon_{K S}\right] \\
& +e^{3}\left[\frac{\partial_{z} \epsilon_{K S}}{V}+\frac{\partial_{z} V}{2 V^{2}} \gamma_{0} \epsilon_{K S}-\frac{\partial_{x} V}{2 V^{2}} \gamma_{3} \gamma_{1}\left(\mathbf{1}-\gamma_{0}\right) \epsilon_{K S}+\frac{\partial_{y} V}{2 V^{2}} \gamma_{2} \gamma_{3}\left(\mathbf{1}-\gamma_{0}\right) \epsilon_{K S}\right]
\end{aligned}
$$

The (spatial) Clifford algebra is spanned by $\mathbf{1}, \gamma_{1}, \gamma_{2}, \gamma_{3}, \gamma_{1} \gamma_{2}, \gamma_{2} \gamma_{3}, \gamma_{3} \gamma_{1}$ and $\gamma_{1} \gamma_{2} \gamma_{3}$; the one forms are spanned by $e^{0}, e^{1}, e^{2}$ and $e^{3}$. Hence the coefficient of every Clifford-algebra basis element in each line of (11) should separately vanish. From the first line of (11), from the basis element 1 we get the equation

$$
\partial_{t} \epsilon_{K S}=0
$$

and from each of the basis elements $\gamma_{1}, \gamma_{2}$, and $\gamma_{3}$ we get the equation

$$
\gamma_{0} \epsilon_{K S}=\epsilon_{K S}
$$

Equation (12) is the expectation that the Killing spinors supported by a time-independent background are timeindependent. Equation (13), using the form of $\gamma_{0}$ from [16], implies that

$$
\epsilon_{K S} \sim\left(\begin{array}{l}
c \\
c
\end{array}\right)
$$

where $c$ is a constant two-component spinor. At this stage, $\epsilon_{K S}$ can be multiplied by a overall function of space. From the second line of (11), the coefficients of the basis elements $\gamma_{1} \gamma_{2}, \gamma_{3} \gamma_{1}$ vanish due to (13) and the equation from the basis element 1 fixes the $x$-dependence of the overall factor. Similarly, the $y$ and $z$-dependence are fixed from the third and fourth lines of (11) and we obtain

$$
\epsilon_{K S}=\frac{1}{\sqrt{V}}\left(\begin{array}{l}
c \\
c
\end{array}\right)
$$

\section{The anti-Killing spinors of the Majumdar-Papapetrou solution}

Anti-Killing spinors [24], $\epsilon_{A K S}$ are spinor fields which correspond to broken supersymmetries and hence at asymptotic infinity should converge to spinors orthogonal to those of Killing spinor fields. Hence $\epsilon_{A K S}$ is proportional to $\left(\begin{array}{c}c \\ -c\end{array}\right)$ which means

$$
\gamma_{0} \epsilon_{A K S}=-\epsilon_{A K S}, \quad\left(\mathbf{1}-\gamma_{0}\right) \epsilon_{A K S}=2 \epsilon_{A K S} .
$$

At this stage we have

$$
\epsilon_{A K S} \sim\left(\begin{array}{c}
c \\
-c
\end{array}\right)
$$

and we can multiply with any function of space-time. We will choose this factor so that the resulting gravitino satisfies the $\gamma$-traceless gauge condition, which is the gauge condition employed throughout this paper. That is

$$
\begin{aligned}
0= & \gamma^{\mu} \hat{\mathcal{D}}_{\mu} \epsilon_{A K S} \\
= & -V \partial_{t} \epsilon_{A K S}-\gamma_{1} \frac{\partial_{x} \epsilon_{A K S}}{V}-\frac{\partial_{x} V}{2 V^{2}} \gamma_{1} \epsilon_{A K S}-\gamma_{2} \frac{\partial_{y} \epsilon_{A K S}}{V} \\
& -\frac{\partial_{y} V}{2 V^{2}} \gamma_{2} \epsilon_{A K S}-\gamma_{3} \frac{\partial_{z} \epsilon_{A K S}}{V}-\frac{\partial_{z} V}{2 V^{2}} \gamma_{3} \epsilon_{A K S}
\end{aligned}
$$

which says the function is time-independent and furthermore fixes the $x, y, z$ dependence to obtain

$$
\epsilon_{A K S}=\frac{1}{\sqrt{V}}\left(\begin{array}{c}
c \\
-c
\end{array}\right) .
$$

\section{Gravitini zero modes due to broken supersymmetries}

One can generate fermion zero modes, i.e., solutions to the fermion equations of motion in the purely bosonic supersymmetric background, by performing a supersymmetry transformation whose supersymmetry parameters are the anti-Killing spinors; note that the anti-Killing spinors do not vanish at infinity and hence they provide for a nontrivial supergauge transformation. From (1), we obtain

$$
\psi_{b s}=\frac{1}{k} \hat{\mathcal{D}} \epsilon_{A K S},
$$


where $b s$ stands for "broken supersymmetries." Using the explicit form for the $\hat{\mathcal{D}}$ operator for the MP background in (8) we have

$$
\begin{aligned}
k \psi_{b s}= & e^{0}\left[\frac{\partial_{x} V}{V^{2}} \gamma_{1}+\frac{\partial_{y} V}{V^{2}} \gamma_{2}+\frac{\partial_{z} V}{V^{2}} \gamma_{3}\right] \epsilon_{A K S} \\
& +e^{1}\left[-\frac{\partial_{x} V}{V^{2}}-\frac{\partial_{y} V}{V^{2}} \gamma_{1} \gamma_{2}+\frac{\partial_{z} V}{V^{2}} \gamma_{3} \gamma_{1}\right] \epsilon_{A K S} \\
& +e^{2}\left[-\frac{\partial_{y} V}{V^{2}}-\frac{\partial_{z} V}{V^{2}} \gamma_{2} \gamma_{3}+\frac{\partial_{x} V}{V^{2}} \gamma_{1} \gamma_{2}\right] \epsilon_{A K S} \\
& +e^{3}\left[-\frac{\partial_{z} V}{V^{2}}-\frac{\partial_{x} V}{V^{2}} \gamma_{3} \gamma_{1}+\frac{\partial_{y} V}{V^{2}} \gamma_{2} \gamma_{3}\right] \epsilon_{A K S}
\end{aligned}
$$

From the above equation, we see that the gravitini field, for the fermion zero mode associated with broken supersymmetries, is a spatial Clifford-algebra element multiplying a constant spinor and also a one-form. This motivates the ansatz in the next section.

\section{THE EQUATIONS FOR GRAVITINI ZERO MODES}

In this section, we will present the generic ansatz for the fermions and set up the fermion equations of motion for this ansatz. We will also present the gauge condition and impose the gauge conditions on the equations of motion to arrive at the final set of equations which need to be solved.

\section{A. An ansatz for the gravitini}

The four dimensional gravitino field $\psi$ consists of four Dirac spinors and as such has $4 \times 8$ real functions, where the 8 is the number of real functions associated to a Dirac spinor field. We will think of the gravitino field as taking values in the Clifford algebra and acting on the constant spinor $\left(\begin{array}{c}c \\ -c\end{array}\right)$, generalizing the fermion zero mode due to broken supersymmetry (19). In our ansatz the 32 real functions associated to $\psi$ will be organized again as $4 \times 8$ but now 8 is the number of real functions associated to a spatial Clifford algebra field:

$$
\begin{aligned}
\psi_{\text {gen }}= & e^{0}\left[h_{0} \mathbf{1}+h_{1} \gamma_{1}+h_{2} \gamma_{2}+h_{3} \gamma_{3}+h_{12} \gamma_{1} \gamma_{2}+h_{23} \gamma_{2} \gamma_{3}+h_{31} \gamma_{3} \gamma_{1}+h_{123} \gamma_{1} \gamma_{2} \gamma_{3}\right] \epsilon_{0} \\
& +e^{1}\left[f_{1} \mathbf{1}+f_{11} \gamma_{1}+f_{12} \gamma_{2}+f_{13} \gamma_{3}+f_{112} \gamma_{1} \gamma_{2}+f_{123} \gamma_{2} \gamma_{3}-f_{113} \gamma_{3} \gamma_{1}+f_{1123} \gamma_{1} \gamma_{2} \gamma_{3}\right] \epsilon_{0} \\
& +e^{2}\left[f_{2} \mathbf{1}+f_{21} \gamma_{1}+f_{22} \gamma_{2}+f_{23} \gamma_{3}-f_{221} \gamma_{1} \gamma_{2}+f_{223} \gamma_{2} \gamma_{3}+f_{231} \gamma_{3} \gamma_{1}+f_{2123} \gamma_{1} \gamma_{2} \gamma_{3}\right] \epsilon_{0} \\
& +e^{3}\left[f_{3} \mathbf{1}+f_{31} \gamma_{1}+f_{32} \gamma_{2}+f_{33} \gamma_{3}+f_{312} \gamma_{1} \gamma_{2}-f_{332} \gamma_{2} \gamma_{3}+f_{331} \gamma_{3} \gamma_{1}+f_{3123} \gamma_{1} \gamma_{2} \gamma_{3}\right] \epsilon_{0} .
\end{aligned}
$$

where $\epsilon_{0}=\left(\begin{array}{c}c \\ -c\end{array}\right)$ is the constant spinor that the anti-Killing spinor field asymptotes to at spatial infinity. It will be useful to define the following vectors in $\mathbf{R}^{3}$ :

$$
\vec{h}=\left(h_{1}, h_{2}, h_{3}\right), \quad \vec{f}=\left(f_{1}, f_{2}, f_{3}\right), \quad \overrightarrow{f_{B}}=\left(f_{221}, f_{332}, f_{113}\right)
$$

$\overrightarrow{f_{C}}=\left(f_{331}, f_{112}, f_{223}\right), \quad \vec{h}_{B}=\left(h_{23}, h_{31}, h_{12}\right)$,

$\overrightarrow{f_{D}}=\left(f_{23}-f_{32}, f_{31}-f_{13}, f_{12}-f_{21}\right)$,

$\overrightarrow{f_{E}}=\left(f_{1123}, f_{2123}, f_{3123}\right)$

It is useful to record, what values the ansatz functions take in $\psi_{b s}$. Comparing (19) and (20), we see

$$
\vec{h}=-\vec{f}=-\overrightarrow{f_{B}}=-\overrightarrow{f_{C}}=\frac{1}{k} \frac{\vec{\nabla} V}{V^{\frac{5}{2}}} \quad \text { for } \psi_{b s}
$$

and all other ansatz functions vanish. The vector field $\frac{\vec{\nabla} V}{V^{\frac{5}{2}}}$ has the right boundary conditions both at asymptotic infinity, where it vanishes as $\mathcal{O}\left(r^{-2}\right)$, and is regular at each of the horizons, where it vanishes as $\mathcal{O}\left(r^{\frac{1}{2}}\right) ; r=0$ is the location of the black hole horizon.

\section{B. Rarita-Schwinger equation in the MP background}

We plug in the ansatz (20) into the Rarita-Schwinger equation in the MP background:

$$
\gamma \wedge d \psi+\gamma \wedge \omega F \wedge \psi=0
$$

where $\omega F$ is defined in (8). Each of the terms in the left hand side above is a three-form taking values in the spatial Clifford algebra. For any such Clifford-algebra valued three-form $\mathcal{E}$, introduce the following notation:

$$
\begin{aligned}
\mathcal{E}= & e^{0} \wedge e^{1} \wedge e^{2}\left[(\mathcal{E})_{012}^{\mathbf{1}} \mathbf{1}+(\mathcal{E})_{012}^{\gamma_{1} \gamma_{1}}+\ldots\right] \\
& +e^{0} \wedge e^{2} \wedge e^{3}\left[(\mathcal{E})_{023}^{\mathbf{1}} \mathbf{1}+(\mathcal{E})_{023}^{\gamma_{1} \gamma_{1}}+\ldots\right] \\
& +e^{0} \wedge e^{3} \wedge e^{1}\left[(\mathcal{E})_{031}^{\mathbf{1}} \mathbf{1}+(\mathcal{E})_{031}^{\gamma_{1}} \gamma_{1}+\ldots\right] \\
& +e^{1} \wedge e^{2} \wedge e^{3}\left[(\mathcal{E})_{123}^{\mathbf{1}} \mathbf{1}+(\mathcal{E})_{123}^{\gamma_{1} \gamma_{1}}+\ldots\right]
\end{aligned}
$$

There are 32 linear partial differential equations that result from (23). It turns out that they split into two decoupled sectors. The first sector consists of 16 ansatz functions: the twelve functions of $\vec{h}, \vec{f}, \overrightarrow{f_{B}}, \overrightarrow{f_{C}}$ and $h_{123}, f_{123}, f_{231}, f_{312}$. These 16 ansatz functions are coupled into a set of 16 linear partial differential equations which are given in the Appendix A 1. The second decoupled sector consists of the remaining 16 ansatz functions which are coupled into a 
set of 16 linear partial differential equations which are given in the Appendix A 2.

We will work with the $\gamma$-traceless gauge condition $\gamma^{\mu} \psi_{\mu}=0$. The $\gamma$-traceless gauge condition is very basic to the structure of gravitini fields. In the flat-space limit of a four dimensional supergravity theory, the gravitino field $\psi_{\mu}$ contains fields in the $\left(1, \frac{1}{2}\right) \oplus\left(\frac{1}{2}, 1\right)$ representations. To obtain states with only helicities $\pm \frac{3}{2}$ [25], one needs to eliminate the spin- $\frac{1}{2}$ bits of the gravitino field $\psi_{\mu}$, which is achieved by imposing the $\gamma$-traceless gauge condition $\gamma^{\mu} \psi_{\mu}=0$. These kind of considerations generalize to the situation when one is in the background of a nontrivial geometry in supergravity $[26,27]$ and is solving linearized equations of motion, which is the situation in this paper. More recently [28] it has been shown that the $\gamma$-traceless condition is the correct gauge condition for the RaritaSchwinger field in supergravity that completes the unimodular gauge choice for the metric, $-\operatorname{det} g_{\mu \nu}=1$, a gauge choice recognized by Albert Einstein as early as 1916 as a preferred gauge choice in classical gravity [29]. For us, the $\gamma$-traceless gauge condition is preferred due to it being a simple algebraic constraint and also because we would like to maintain a continuity with the work we wish to go beyond [9] and which uses this gauge condition.

The $\gamma$-traceless gauge condition for the ansatz (20) results in the following equations:

$$
\begin{aligned}
& h_{123}-f_{123}-f_{231}-f_{312}=0, \vec{h}-\vec{f}+\overrightarrow{f_{B}}+\overrightarrow{f_{C}}=0 \\
&-\vec{h}_{B}-\overrightarrow{f_{D}}+\overrightarrow{f_{E}}=0, \quad-h_{0}+f_{11}+f_{22}+f_{33}=0
\end{aligned}
$$

Note that the first line is a condition on the ansatz functions in the first decoupled sector and the second line is a condition for the second decoupled sector. Thus $\gamma$-traceless condition is consistent with the separation of the ansatz functions into two decoupled sectors.

We now impose the gauge condition (25) on the equations for the first decoupled sector in A 1 . We eliminate $h_{123}$ and $\vec{h}$ and obtain the following set of partial differential equations for $\vec{f}, \vec{f}_{B}, \vec{f}_{C}, f_{123}, f_{231}, f_{312}$.

$$
\begin{gathered}
\vec{\nabla} \times\left[\frac{\vec{f}_{B}+\vec{f}_{C}}{\sqrt{V}}\right]=0 . \\
\partial_{x}\left[\frac{f_{1}-f_{C 1}}{\sqrt{V}}\right]+\partial_{y}\left[\frac{f_{2}-f_{B 2}}{\sqrt{V}}\right]=0 \\
\partial_{y}\left[\frac{f_{2}-f_{C 2}}{\sqrt{V}}\right]+\partial_{z}\left[\frac{f_{3}-f_{B 3}}{\sqrt{V}}\right]=0 \\
\partial_{z}\left[\frac{f_{3}-f_{C 3}}{\sqrt{V}}\right]+\partial_{x}\left[\frac{f_{1}-f_{B 1}}{\sqrt{V}}\right]=0 .
\end{gathered}
$$

$$
\begin{aligned}
& \partial_{x}\left[\frac{f_{3}-f_{B 3}}{\sqrt{V}}\right]+\partial_{y}\left[\frac{A_{1}}{\sqrt{V}}\right]=0 \\
& \partial_{y}\left[\frac{f_{1}-f_{B 1}}{\sqrt{V}}\right]+\partial_{z}\left[\frac{A_{2}}{\sqrt{V}}\right]=0 \\
& \partial_{z}\left[\frac{f_{2}-f_{B 2}}{\sqrt{V}}\right]+\partial_{x}\left[\frac{A_{3}}{\sqrt{V}}\right]=0 \text {. } \\
& \partial_{y}\left[\frac{f_{3}-f_{C 3}}{\sqrt{V}}\right]-\partial_{x}\left[\frac{A_{2}}{\sqrt{V}}\right]=0 \\
& \partial_{z}\left[\frac{f_{1}-f_{C 1}}{\sqrt{V}}\right]-\partial_{y}\left[\frac{A_{3}}{\sqrt{V}}\right]=0 \\
& \partial_{x}\left[\frac{f_{2}-f_{C 2}}{\sqrt{V}}\right]-\partial_{z}\left[\frac{A_{1}}{\sqrt{V}}\right]=0 . \\
& \frac{1}{\sqrt{V}} \vec{\nabla} \cdot\left[\frac{\overrightarrow{f_{B}}+\vec{f}_{C}}{\sqrt{V}}\right]+4 \vec{F} \cdot\left(\vec{f}+\vec{f}_{B}+\vec{f}_{C}\right)=0 \\
& \frac{1}{\sqrt{V}} \partial_{y}\left[\frac{f_{3}-f_{B 3}}{\sqrt{V}}\right]-\frac{1}{\sqrt{V}} \partial_{z}\left[\frac{f_{2}-f_{C 2}}{\sqrt{V}}\right]-\frac{1}{\sqrt{V}} \partial_{x}\left[\frac{A_{1}}{\sqrt{V}}\right] \\
& +4 F_{2}\left[f_{3}-f_{B 3}+f_{C 3}\right]-4 F_{3}\left[f_{2}+f_{B 2}-f_{C 2}\right] \\
& -4 F_{1}\left[A_{1}-f_{123}\right]=0 \\
& \frac{1}{\sqrt{V}} \partial_{z}\left[\frac{f_{1}-f_{B 1}}{\sqrt{V}}\right]-\frac{1}{\sqrt{V}} \partial_{x}\left[\frac{f_{3}-f_{C 3}}{\sqrt{V}}\right]-\frac{1}{\sqrt{V}} \partial_{y}\left[\frac{A_{2}}{\sqrt{V}}\right] \\
& +4 F_{3}\left[f_{1}-f_{B 1}+f_{C 1}\right]-4 F_{1}\left[f_{3}+f_{B 3}-f_{C 3}\right] \\
& -4 F_{2}\left[A_{2}-f_{231}\right]=0 \\
& \frac{1}{\sqrt{V}} \partial_{x}\left[\frac{f_{2}-f_{B 2}}{\sqrt{V}}\right]-\frac{1}{\sqrt{V}} \partial_{y}\left[\frac{f_{1}-f_{C 1}}{\sqrt{V}}\right]-\frac{1}{\sqrt{V}} \partial_{z}\left[\frac{A_{3}}{\sqrt{V}}\right] \\
& +4 F_{1}\left[f_{2}-f_{B 2}+f_{C 2}\right]-4 F_{2}\left[f_{1}+f_{B 1}-f_{C 1}\right] \\
& -4 F_{3}\left[A_{3}-f_{312}\right]=0
\end{aligned}
$$

There are sixteen (one scalar, two vectors and one second-rank tensor) equations for twelve variables. We will address the solutions to these equations in the next section. We will not write the analogous sixteen equations for the second decoupled sector here since in this paper, we will restrict ourselves to a search for extra fermion zero modes only in the first decoupled sector. We can think of the solutions we will obtain as having zero for the values of all the ansatz functions in the second decoupled sector.

\section{GRAVITINI ZERO MODES BEYOND BROKEN SUPERSYMMETRIES}

We will study known solutions before we start to solve the equations in the first decoupled sector viz. (26)-(39). First we will consider the fermion zero mode due to broken 
supersymmetries $\psi_{b s}$ given in (22). The fact that $\vec{f}=\vec{f}_{B}=$ $\vec{f}_{C}$ together with the vanishing of $f_{123}, f_{231}, f_{312}$ means that $\psi_{b s}$ solves Eqs. (27)-(35). Those facts together with the fact that the vectors $\vec{f}, \vec{f}_{B}, \vec{f}_{C}$ are proportional to the gradient $\vec{\nabla} V$ means that $\psi_{b s}$ also solves (26) and (37)-(39). Finally we are left with (36) which can be directly verified. This check that $\psi_{b s}$ solves the equations we have derived constitutes a check on the validity of the equations.

Now we will solve the Eqs. (26)-(39). These are a system of coupled first order partial differential equations, to be solved on $\mathbf{R}^{3}$. Using (26), we can write the vector field there in terms of a function $\mathcal{G}(x, y, z)$ :

$$
\overrightarrow{f_{B}}+\overrightarrow{f_{C}}=\sqrt{V} \vec{\nabla} \mathcal{G}
$$

Then, we consider the sum of the equations (27), (28), (29)

$$
\vec{\nabla} \cdot\left[\frac{2 \vec{f}-\vec{f}_{B}-\vec{f}_{C}}{\sqrt{V}}\right]=0,
$$

which together with (40) allows us to write the vector field $\vec{f}$

$$
\frac{\vec{f}}{\sqrt{V}}=\frac{\vec{\nabla} \mathcal{G}}{2}+\frac{\overrightarrow{\mathcal{A}}}{2}
$$

where $\overrightarrow{\mathcal{A}}$ is a divergence-free vector field in $\mathbf{R}^{3}$. We have thus far used a subset of the equations to parametrize the vector fields $\vec{f}$ and $\vec{f}_{B}+\vec{f}_{C}$ in terms of a scalar $\mathcal{G}$ and a divergence-free vector field $\overrightarrow{\mathcal{A}}$. These vector fields are also the only ones which appear in the scalar equation (36), which after using (40), (42), becomes

$$
\nabla^{2} \mathcal{G}+3 \frac{\vec{\nabla} V}{V} \cdot \vec{\nabla} \mathcal{G}+\frac{\vec{\nabla} V}{V} \cdot \overrightarrow{\mathcal{A}}=0
$$

At this stage, we note that for $\psi_{b s}(22), \mathcal{G}_{b s}=\frac{1}{V^{2}}$ and $\overrightarrow{\mathcal{A}}_{b s}=0$. This suggests the following way of solving (43):

$$
\mathcal{G}=-\frac{C}{V^{2}}, \quad \vec{\nabla} V \cdot \overrightarrow{\mathcal{A}}=0
$$

where $\overrightarrow{\mathcal{A}}$ is now a nonzero divergence-free vector field, $C$ is an integration constant. This choice for $\mathcal{G}$ ensures that the first summand that contributes to $\vec{f}$ in (42) has the right regularity properties, both at asymptotic infinity and at any of the horizons. We now need to solve for $\overrightarrow{\mathcal{A}}$ with the right regularity properties.

Using the fact that a divergence-free vector field in three dimensions can be written as a vector cross product of two gradient vector fields [30], together with the restriction on the divergence-free vector field coming from (44), we can take

$$
\overrightarrow{\mathcal{A}}=\vec{\nabla}\left[-\frac{1}{V^{2}}\right] \times \vec{\nabla} \mathcal{H}
$$

where $\mathcal{H}$ is a function. We have chosen one of the gradient vector fields to be $\vec{\nabla}\left[-\frac{1}{V^{2}}\right]$. This solves (44) and already has the required regularity properties at asymptotic infinity as well as at the horizons which means that $\mathcal{H}$ can be any function such that $\vec{\nabla} \mathcal{H}$ is $\mathcal{O}(1)$ at asymptotic infinity and is regular at any of the horizons. We will start with the following choice

$$
\mathcal{H}(x, y, z)=D_{1} x
$$

where $D_{1}$ is an integration constant. We now have

$$
\vec{f}=C\left[\frac{\vec{\nabla} V}{V^{\frac{5}{2}}}\right]+D_{1}\left[\frac{\vec{\nabla} V}{V^{\frac{5}{2}}} \times(1,0,0)\right] .
$$

We have thus far completely determined $\vec{f}$ and the chosen $\mathcal{G}$ in (44) determines the sum $\vec{f}_{B}+\vec{f}_{C}$ (40) but the difference is still undetermined, which we parametrize via the vector field $\vec{L}$ as

$$
\frac{\vec{f}_{B}}{\sqrt{V}}=C\left[\frac{\vec{\nabla} V}{V^{3}}\right]-\vec{L}, \quad \frac{\vec{f}_{C}}{\sqrt{V}}=C\left[\frac{\vec{\nabla} V}{V^{3}}\right]+\vec{L} .
$$

This vector field should be required to have the correct regularity properties. Substituting (47), (48) into the equations (27)-(29), we obtain equations for the components of $\vec{L}$

$$
\begin{aligned}
& -\partial_{x} L_{1}+\partial_{y} L_{2}=-D_{1} \partial_{y}\left[\frac{\partial_{z} V}{V^{3}}\right] \\
& -\partial_{y} L_{2}+\partial_{z} L_{3}=0 \\
& -\partial_{z} L_{3}+\partial_{x} L_{1}=D_{1} \partial_{z}\left[\frac{\partial_{y} V}{V^{3}}\right]
\end{aligned}
$$

and the following is one solution to these equations

$L_{1}=0, \quad L_{2}=-D_{1}\left[\frac{\partial_{z} V}{V^{3}}\right] \quad L_{3}=-D_{1}\left[\frac{\partial_{y} V}{V^{3}}\right]$.

Thus far, we have managed to obtain the ansatz functions in $\vec{f}, \vec{f}_{B}, \vec{f}_{C}$. We have not yet solved for the ansatz functions $f_{123}, f_{231}, f_{312}$. When we substitute the results (47), (48), (50) into the yet-to-be-solved Eqs. (30)-(35), we obtain equations which can be readily solved

$$
A_{1}=2 D_{1} \frac{\partial_{x} V}{V^{\frac{5}{2}}}, \quad A_{2}=0, \quad A_{3}=0
$$

which gives

$$
\begin{aligned}
& f_{123}=-D_{1} \frac{\partial_{x} V}{V^{\frac{5}{2}}}, \quad f_{231}=D_{1} \frac{\partial_{x} V}{V^{\frac{5}{2}}}, \\
& f_{312}=D_{1} \frac{\partial_{x} V}{V^{\frac{5}{2}}} .
\end{aligned}
$$

We have obtained all the ansatz functions in this sector, but we have not used the Eqs. (37), (38), (39). It turns out that 
our solution solves these equations also. Hence we have the solution

$$
\begin{aligned}
\vec{f} & =\left(C \frac{\partial_{x} V}{V^{\frac{5}{2}}}, C \frac{\partial_{y} V}{V^{\frac{5}{2}}}+D_{1} \frac{\partial_{z} V}{V^{\frac{5}{2}}}, C \frac{\partial_{z} V}{V^{\frac{5}{2}}}-D_{1} \frac{\partial_{y} V}{V^{\frac{5}{2}}}\right) \\
\vec{f}_{B} & =\left(C \frac{\partial_{x} V}{V^{\frac{5}{2}}}, C \frac{\partial_{y} V}{V^{\frac{5}{2}}}+D_{1} \frac{\partial_{z} V}{V^{\frac{5}{2}}}, C \frac{\partial_{z} V}{V^{\frac{5}{2}}}+D_{1} \frac{\partial_{y} V}{V^{\frac{5}{2}}}\right) \\
\vec{f}_{C} & =\left(C \frac{\partial_{x} V}{V^{\frac{5}{2}}}, C \frac{\partial_{y} V}{V^{\frac{5}{2}}}-D_{1} \frac{\partial_{z} V}{V^{\frac{5}{2}}}, C \frac{\partial_{z} V}{V^{\frac{5}{2}}}-D_{1} \frac{\partial_{y} V}{V^{\frac{5}{2}}}\right) \\
f_{123} & =-D_{1} \frac{\partial_{x} V}{V^{\frac{5}{2}}}, \quad f_{231}=D_{1} \frac{\partial_{x} V}{V^{\frac{5}{2}}}, \quad f_{312}=D_{1} \frac{\partial_{x} V}{V^{\frac{5}{2}}} .
\end{aligned}
$$

We can quickly generalize this solution as follows. A more general choice for $\mathcal{H}$ that goes beyond (46) but satisfies the required regularity conditions at asymptotic infinity and at the horizons is

$$
\mathcal{H}=D_{1} x+D_{2} y+D_{3} z
$$

which leads to

$$
\overrightarrow{\mathcal{A}}=\vec{\nabla}\left[-\frac{1}{V^{2}}\right] \times \vec{D} \quad \text { where } \vec{D} \equiv\left(D_{1}, D_{2}, D_{3}\right)
$$

One can repeat along the lines of the above analysis and obtain the more general solution

$$
\begin{aligned}
\vec{f} & =C \frac{\vec{\nabla} V}{V^{\frac{5}{2}}}+\frac{\vec{\nabla} V}{V^{\frac{5}{2}}} \times \vec{D} \\
\vec{f}_{B} & =C \frac{\vec{\nabla} V}{V^{\frac{5}{2}}}+\left(D_{2} \frac{\partial_{z} V}{V^{\frac{5}{2}}}+D_{3} \frac{\partial_{y} V}{V^{\frac{5}{2}}}, D_{3} \frac{\partial_{x} V}{V^{\frac{5}{2}}}+D_{1} \frac{\partial_{z} V}{V^{\frac{5}{2}}}, D_{1} \frac{\partial_{y} V}{V^{\frac{5}{2}}}+D_{2} \frac{\partial_{x} V}{V^{\frac{5}{2}}}\right) \\
\vec{f}_{C} & =C \frac{\vec{\nabla} V}{V^{\frac{5}{2}}}-\left(D_{2} \frac{\partial_{z} V}{V^{\frac{5}{2}}}+D_{3} \frac{\partial_{y} V}{V^{\frac{5}{2}}}, D_{3} \frac{\partial_{x} V}{V^{\frac{5}{2}}}+D_{1} \frac{\partial_{z} V}{V^{\frac{5}{2}}}, \quad D_{1} \frac{\partial_{y} V}{V^{\frac{5}{2}}}+D_{2} \frac{\partial_{x} V}{V^{\frac{5}{2}}}\right) \\
f_{123} & =-D_{1} \frac{\partial_{x} V}{V^{\frac{5}{2}}}+D_{2} \frac{\partial_{y} V}{V^{\frac{5}{2}}}+D_{3} \frac{\partial_{z} V}{V^{\frac{5}{2}}} \\
f_{231} & =D_{1} \frac{\partial_{x} V}{V^{\frac{5}{2}}}-D_{2} \frac{\partial_{y} V}{V^{\frac{5}{2}}}+D_{3} \frac{\partial_{z} V}{V^{\frac{5}{2}}} \\
f_{312} & =D_{1} \frac{\partial_{x} V}{V^{\frac{5}{2}}}+D_{2} \frac{\partial_{y} V}{V^{\frac{5}{2}}}-D_{3} \frac{\partial_{z} V}{V^{\frac{5}{2}}}
\end{aligned}
$$

\section{A. The supercharge}

The supercharge of a solution is given [9] by

$$
Q=-\frac{i}{k} \oint_{S_{\infty}^{2}} \gamma_{5} \gamma \wedge \psi
$$

which for the solution in (56) computes to

$$
Q=2 i k M\left(\gamma^{i} D_{i} \gamma_{5}-C \gamma_{0}\right) \epsilon_{0} .
$$

The integration constant $C$ is associated with the solution from broken supersymmetries. While the solutions accompanying the three integration constants $D_{1}, D_{2}, D_{3}$, are new solutions to the fermionic equations of motion. We have thus obtained extra fermion zero mode solutions. Since the integration constants $D_{1}, D_{2}, D_{3}$, appear in the supercharge, they are physical and not some gauge artifacts.

\section{B. Counting the zero modes [31]}

Pure gauge solutions have zero supercharge and hence should not be counted. Now we ask which among the solutions we have obtained have vanishing supercharge. Our solutions are parametrized by the four integration constants $C, D_{1}, D_{2}, D_{3}$ and the constant two component complex spinor $c$ that is in $\epsilon_{0}$. Finding the pure gauge solutions thus amounts to asking if we can adjust the values of $C, D_{1}, D_{2}, D_{3}$ and the constant spinor $c$ in such a way that the supercharge $Q$ given in Eq. (58) vanishes.

First we study the case $D_{2}=D_{3}=0$. Then analyzing (58) shows that for

$$
C=D_{1}, \quad c=\left(\begin{array}{l}
p \\
p
\end{array}\right)
$$

and for

$$
C=-D_{1}, \quad c=\left(\begin{array}{c}
p \\
-p
\end{array}\right)
$$

we obtain $Q=0$. We can then generalize this analysis to show that for every vector $\vec{D}$, we can find a specific value for the integration constant $C$ and a specific constant spinor 
$c$ so that the supercharge $Q$ vanishes. What this means is that effectively the number of independent zero modes carrying supercharge will be parametrized by $C$ and the constant spinor $c$, which gives four zero modes.

Thus the extra gravitini zero mode solutions that we have obtained in this paper (56), after discounting the pure gauge solutions, constitute four independent zero modes.

\section{CONCLUSION AND FUTURE DIRECTIONS}

In this paper, we have achieved the goal we set for ourselves, and which we outlined in the introduction. We have provided a set up to study the fermionic equations of motion in the background of purely bosonic supersymmetric solutions. We have done this in particular for the Rarita-Schwinger equations for the gravitini of four dimensional $\mathcal{N}=2$ supergravity and for the MajumdarPapapetrou solutions. We have obtained solutions to those equations beyond the ones corresponding to the broken supersymmetries. We have thus obtained some extra fermion zero mode solutions. Even if we have already mentioned this in the introduction, we again reiterate here that we could not have obtained these extra zero mode solutions without going through the methods of this paper, i.e., by explicitly computing the fermion equations of motion for a fairly big ansatz and recasting them into the electrodynamicslike equations. All earlier solutions available in the literature, the zero modes corresponding to broken supersymmetries, can be obtained and indeed were obtained, without having to explicitly solve the fermion equations of motion. This is the main achievement of this paper. But, clearly we have not obtained all the solutions to the equations and hence there are more of these extra fermion zero modes, which is a natural study to take up. One should include the equations coming from the second decoupled sector A 2. We hope to make progress on this front [23].

The solutions we have obtained in this paper are at the linear order in the fermion parameter $c$ [see (20)] for the gravitini field and are at zeroth order in fermion parameter for the bosonic fields viz. the metric and the gauge field. Having found the gravitini one then can proceed with an iterative procedure and calculate the backreaction of the gravitini on the metric and the gauge field. This should lead to corrections to the original Majumdar-Papapetrou solution in the metric and the gauge fields which are of second order in the fermion parameter $c$. This in turn induces additional corrections to the gravitini which would be of third order in the fermion parameter. Such an iterative procedure stops after the fourth order in the fermion parameter $c$ and leads to an exact solution to the full supergravity equations of motion. We would like to obtain and study this full nonlinear backreacted solution, starting from our solution (53), in subsequent work [23]. Although it must be said that such a backreacted solution obtained from the linear solution corresponding to broken super symmetries [for $D_{1}=D_{2}=D_{3}=0$ in (53)] already exists in the literature; see [9]. Again, this was obtained not by following the above outlined much-involved iterative procedure of solving the full supergravity equations of motion, but rather by simply performing a finite supersymmetry transformation, or in other words, an iteration of (or an exponentiation) the infinitesimal supersymmetry transformation on the zeroth order bosonic solution. We give here only the metric of the full nonlinear solution corresponding to broken supersymmetries

$$
\begin{aligned}
g= & {\left[V^{-2}+\frac{5}{2 V^{8}} \partial_{i} V \partial_{i} V\left(c^{\dagger} c\right)\left(c^{\dagger} c\right)\right] d t^{2} } \\
& +\frac{4}{V^{3}} \epsilon_{i j k} \partial_{i} V c^{\dagger} \sigma_{j} c d x^{k} d t-V^{2} \delta_{i j} d x^{i} d x^{j} \\
& -V^{-4}\left(V \partial_{i j}^{2} V-\frac{11}{3} \partial_{i} V \partial_{j} V+\frac{5}{3} \delta_{i j} \partial_{k} V \partial_{k} V\right) \\
& \times\left(c^{\dagger} c\right)\left(c^{\dagger} c\right) d x^{i} d x^{j} ;
\end{aligned}
$$

the gauge field which is quartic in the fermion parameter can be found in Eq. (2.34c) and the gravitini which are cubic in the fermion parameter can be found in Eq. (2.34d) of [9]. We aim to obtain the full nonlinear solution corresponding to the solution (53), that is, the nonzero $D_{1}, D_{2}, D_{3}$ generalization of (61), in [23].

Even if we will postpone the full study of the change in the metric and its causal structure due to the fermionic contributions to the future work, we can already say that the full nonlinear solutions will have the proper regularity properties at all the horizons as well as asymptotic infinity. This is because we have incorporated that at various stages of our analysis (see (45) and the subsequent paragraph), taking our cues from the structure of the solution due to broken super symmetries, which is regular at the horizons and at asymptotic infinity. The full nonlinear solution that we hope to achieve in the future has other applications: in the more general case of $\mathcal{N}=2$ supergravity coupled to vector multiplets, can verify conjectures pertaining to black hole physics given in [32].

The methods and techniques of this paper can be applied for other purely bosonic supersymmetric solutions of four dimensional $\mathcal{N}=2$ supergravity such as the most general solutions of [6]. They can also be applied (i) first for the Denef-Bates multicenter solutions [18] in the context of four dimensional $\mathcal{N}=2$ supergravity theory that includes vector multiplets and eventually (ii) to multicenter solutions in the context of four dimensional $\mathcal{N}=4$ supergravity.

The structure of five dimensional $\mathcal{N}=2$ pure supergravity is very similar to its four dimensional analog. The field content is also similar, one-forms valued in functions and spinors. A complete classification of purely bosonic solutions to this theory already exists [33]. Very interesting bosonic solutions exist, such as a Beckenridge-Myers-PeetVafa [34] like rotating black hole and the supersymmetric 
black ring [35]. One hopes to develop a theory of all fermion zero mode solutions to minimal supergravity in five dimensions.

We also need to explore the consequences of our solutions (56) to black hole physics. Questions such as the relation between the fermion zero modes to the two black hole solution and the fermion zero modes of the two individual single black holes need to be answered.

In this paper, we have studied the linearized equations of motion for the gravitini fields around the black hole, with the aim of answering questions pertaining to black hole physics (especially with respect to the microscopics of black hole entropy). Another problem where one considers linearized equations of motion around black holes, is that of stability of black holes. One considers perturbations of various fields on the black hole solutions and to address the stability of the black hole against these perturbations, one has to study the linearized equations. Other issues related to stability are of quasinormal modes and quasinormal frequencies of various fields where it is needed to impose a special class of boundary conditions at the horizon and at asymptotic infinity and obtain solutions to linearized equations for the fields $[36,37]$. The methods and the results of this paper, we believe, could be adapted, to address stability of extreme Reissner-Nordstrom black holes against gravitini perturbations and probably compute the gravitini quasinormal modes and frequencies. Studies of linearized solutions for gravitino perturbations to various black holes have appeared in the literature: [38-41]. Some of these studies feature a decoupling of the perturbations into two sectors, much like what we see in this paper. We hope to take up the study of stability of the MajumdarPapapetrou black holes, especially to gravitino perturbations, in future work.

\section{ACKNOWLEDGMENTS}

We would like to thank Prof. Ashoke Sen for crucial discussions and guidance through out the course of this project. We would like to thank Taniya Mandal for discussions and collaboration at the early stages of the project. C. N. G. is very thankful to the High-EnergySection, ICTP, Trieste and in particular Prof. Bobby Acharya and Prof. Atish Dabholkar, for a visit to the ICTP in whose stimulating atmosphere a good part of this work was done.

\section{APPENDIX A: FERMION EQUATIONS}

Let $\mathcal{F E}$ denote the Clifford-algebra valued three-form equation (23) and let $\vec{F}=\frac{\vec{\nabla} V}{2 V^{2}}$.

\section{The first decoupled sector}

The three equations $\mathcal{F E}_{012}^{1}, \mathcal{F E}_{023}^{1}$, and $\mathcal{F E}_{031}^{1}$ can be combined nicely into the following vector equation:

$$
\vec{\nabla} \times\left[\frac{\vec{h}-\vec{f}}{V^{\frac{1}{2}}}\right]=0
$$

The nine equations $\quad \mathcal{F E}_{012}^{\gamma_{1} \gamma_{2}}, \quad \mathcal{F E}_{012}^{\gamma_{2} \gamma_{3}}, \quad \mathcal{F} \mathcal{E}_{012}^{\gamma_{3} \gamma_{1}}$, $\mathcal{F} \mathcal{E}_{023}^{\gamma_{1} \gamma_{2}} \ldots \mathcal{F} \mathcal{E}_{031}^{\gamma_{3} \gamma_{1}}$ form a rank two Cartesian tensor worth of equations:

$$
\begin{gathered}
\partial_{x}\left[\frac{h_{1}+f_{B 1}}{\sqrt{V}}\right]+\partial_{y}\left[\frac{h_{2}+f_{C 2}}{\sqrt{V}}\right]=0 \\
\partial_{y}\left[\frac{h_{2}+f_{B 2}}{\sqrt{V}}\right]+\partial_{z}\left[\frac{h_{3}+f_{C 3}}{\sqrt{V}}\right]=0 \\
\partial_{z}\left[\frac{h_{3}+f_{B 3}}{\sqrt{V}}\right]+\partial_{x}\left[\frac{h_{1}+f_{C 1}}{\sqrt{V}}\right]=0 . \\
\partial_{x}\left[\frac{h_{3}+f_{C 3}}{\sqrt{V}}\right]+\partial_{y}\left[\frac{h_{123}-f_{123}}{\sqrt{V}}\right]=0 \\
\partial_{y}\left[\frac{h_{1}+f_{C 1}}{\sqrt{V}}\right]+\partial_{z}\left[\frac{h_{123}-f_{231}}{\sqrt{V}}\right]=0 \\
\partial_{z}\left[\frac{h_{2}+f_{C 2}}{\sqrt{V}}\right]+\partial_{x}\left[\frac{h_{123}-f_{312}}{\sqrt{V}}\right]=0 . \\
\partial_{y}\left[\frac{h_{3}+f_{B 3}}{\sqrt{V}}\right]-\partial_{x}\left[\frac{h_{123}-f_{231}}{\sqrt{V}}\right]=0 \\
\partial_{z}\left[\frac{h_{1}+f_{B 1}}{\sqrt{V}}\right]-\partial_{y}\left[\frac{h_{123}-f_{312}}{\sqrt{V}}\right]=0 \\
\partial_{x}\left[\frac{h_{2}+f_{B 2}}{\sqrt{V}]-\partial_{z}\left[\frac{h_{123}-f_{123}}{\sqrt{V}}\right]=0 .}\right.
\end{gathered}
$$

The equation $\mathcal{F E}_{123}^{\gamma_{1} \gamma_{2} \gamma_{3}}$ is the following scalar equation:

$$
\frac{1}{\sqrt{V}} \vec{\nabla} \cdot\left[\frac{\overrightarrow{f_{B}}+\vec{f}_{C}}{\sqrt{V}}\right]+4 \vec{F} \cdot\left(\vec{f}+\vec{f}_{B}+\vec{f}_{C}\right)=0 .
$$

The three equations $\mathcal{F} \mathcal{E}_{123}^{\gamma_{1}}, \mathcal{F} \mathcal{E}_{123}^{\gamma_{2}}, \mathcal{F} \mathcal{E}_{123}^{\gamma_{3}}$ are the following vector equation:

$$
\begin{aligned}
& \frac{1}{\sqrt{V}} \partial_{y}\left[\frac{f_{3}-f_{B 3}}{\sqrt{V}}\right]-\frac{1}{\sqrt{V}} \partial_{z}\left[\frac{f_{2}-f_{C 2}}{\sqrt{V}}\right]-\frac{1}{\sqrt{V}} \partial_{x}\left[\frac{A_{1}}{\sqrt{V}}\right] \\
& \quad+4 F_{2}\left[f_{3}-f_{B 3}+f_{C 3}\right]-4 F_{3}\left[f_{2}+f_{B 2}-f_{C 2}\right] \\
& \quad-4 F_{1}\left[A_{1}-f_{123}\right]=0 \\
& \frac{1}{\sqrt{V}} \partial_{z}\left[\frac{f_{1}-f_{B 1}}{\sqrt{V}}\right]-\frac{1}{\sqrt{V}} \partial_{x}\left[\frac{f_{3}-f_{C 3}}{\sqrt{V}}\right]-\frac{1}{\sqrt{V}} \partial_{y}\left[\frac{A_{2}}{\sqrt{V}}\right] \\
& \quad+4 F_{3}\left[f_{1}-f_{B 1}+f_{C 1}\right]-4 F_{1}\left[f_{3}+f_{B 3}-f_{C 3}\right] \\
& -4 F_{2}\left[A_{2}-f_{231}\right]=0
\end{aligned}
$$




$$
\begin{aligned}
& \frac{1}{\sqrt{V}} \partial_{x}\left[\frac{f_{2}-f_{B 2}}{\sqrt{V}}\right]-\frac{1}{\sqrt{V}} \partial_{y}\left[\frac{f_{1}-f_{C 1}}{\sqrt{V}}\right]-\frac{1}{\sqrt{V}} \partial_{z}\left[\frac{A_{3}}{\sqrt{V}}\right] \\
& \quad+4 F_{1}\left[f_{2}-f_{B 2}+f_{C 2}\right]-4 F_{2}\left[f_{1}+f_{B 1}-f_{C 1}\right]-4 F_{3}\left[A_{3}-f_{312}\right]=0
\end{aligned}
$$

where we have defined

$$
A_{1} \equiv f_{312}+f_{231}, \quad A_{2} \equiv f_{123}+f_{312} \quad A_{3} \equiv f_{231}+f_{123}
$$

\section{The second decoupled sector}

The equation $\mathcal{F E}_{123}^{1}$ is the following scalar equation:

$$
\vec{\nabla} \cdot \vec{f}_{D}+\frac{3}{2} \frac{\vec{\nabla} V \cdot \vec{f}_{D}}{V}=0
$$

The nine equations $\mathcal{F} \mathcal{E}_{012}^{\gamma_{1}}, \mathcal{F} \mathcal{E}_{012}^{\gamma_{2}}, \mathcal{F} \mathcal{E}_{012}^{\gamma_{3}}, \mathcal{F} \mathcal{E}_{023}^{\gamma_{1}} \ldots \mathcal{F} \mathcal{E}_{031}^{\gamma_{3}}$ form a rank two Cartesian tensor worth of equations:

$$
\begin{aligned}
& \frac{1}{\sqrt{V}} \partial_{x}\left[\frac{h_{23}+f_{23}}{\sqrt{V}}\right]+\frac{1}{\sqrt{V}} \partial_{y}\left[\frac{h_{31}-f_{13}}{\sqrt{V}}\right]+4 F_{1} f_{23}-4 F_{2} f_{13}+4 F_{3} h_{12}=0 \\
& \frac{1}{\sqrt{V}} \partial_{y}\left[\frac{h_{31}+f_{31}}{\sqrt{V}}\right]+\frac{1}{\sqrt{V}} \partial_{z}\left[\frac{h_{12}-f_{21}}{\sqrt{V}}\right]+4 F_{2} f_{31}-4 F_{3} f_{21}+4 F_{1} h_{23}=0 \\
& \frac{1}{\sqrt{V}} \partial_{z}\left[\frac{h_{12}+f_{12}}{\sqrt{V}}\right]+\frac{1}{\sqrt{V}} \partial_{x}\left[\frac{h_{23}-f_{32}}{\sqrt{V}}\right]+4 F_{3} f_{12}-4 F_{1} f_{32}+4 F_{2} h_{31}=0 \\
& \frac{1}{\sqrt{V}} \partial_{x}\left[\frac{h_{0}-f_{22}}{\sqrt{V}}\right]+\frac{1}{\sqrt{V}} \partial_{y}\left[\frac{h_{12}+f_{12}}{\sqrt{V}}\right]-4 F_{1} f_{22}+4 F_{2} f_{12}-4 F_{3} h_{31}=0 \\
& \frac{1}{\sqrt{V}} \partial_{y}\left[\frac{h_{0}-f_{33}}{\sqrt{V}}\right]+\frac{1}{\sqrt{V}} \partial_{z}\left[\frac{h_{23}+f_{23}}{\sqrt{V}}\right]-4 F_{2} f_{33}+4 F_{3} f_{23}-4 F_{1} h_{12}=0 \\
& \frac{1}{\sqrt{V}} \partial_{z}\left[\frac{h_{0}-f_{11}}{\sqrt{V}}\right]+\frac{1}{\sqrt{V}} \partial_{x}\left[\frac{h_{31}+f_{31}}{\sqrt{V}}\right]-4 F_{3} f_{11}+4 F_{1} f_{31}-4 F_{2} h_{23}=0 \\
& \frac{1}{\sqrt{V}} \partial_{x}\left[\frac{h_{12}-f_{21}}{\sqrt{V}}\right]-\frac{1}{\sqrt{V}} \partial_{y}\left[\frac{h_{0}-f_{11}}{\sqrt{V}}\right]-4 F_{1} f_{21}+4 F_{2} f_{11}-4 F_{3} h_{23}=0 \\
& \frac{1}{\sqrt{V}} \partial_{y}\left[\frac{h_{23}-f_{32}}{\sqrt{V}}\right]-\frac{1}{\sqrt{V}} \partial_{z}\left[\frac{h_{0}-f_{22}}{\sqrt{V}}\right]-4 F_{2} f_{32}+4 F_{3} f_{22}-4 F_{1} h_{31}=0 \\
& \frac{1}{\sqrt{V}} \partial_{z}\left[\frac{h_{31}-f_{13}}{\sqrt{V}}\right]-\frac{1}{\sqrt{V}} \partial_{x}\left[\frac{h_{0}-f_{33}}{\sqrt{V}}\right]-4 F_{3} f_{13}+4 F_{1} f_{33}-4 F_{2} h_{12}=0
\end{aligned}
$$

The three equations $\mathcal{F E}_{123}^{\gamma_{1} \gamma_{2}}, \mathcal{F} \mathcal{E}_{123}^{\gamma_{2} \gamma_{3}}, \mathcal{F} \mathcal{E}_{123}^{\gamma_{3} \gamma_{1}}$ are

$$
\begin{aligned}
& \frac{1}{\sqrt{V}} \partial_{x}\left[\frac{f_{31}-f_{2123}}{\sqrt{V}}\right]+\frac{1}{\sqrt{V}} \partial_{y}\left[\frac{f_{32}+f_{1123}}{\sqrt{V}}\right]-\frac{1}{\sqrt{V}} \partial_{z}\left[\frac{f_{11}+f_{22}}{\sqrt{V}}\right]+4 F_{1}\left(f_{31}-f_{2123}\right)-4 F_{2}\left(f_{32}+f_{1123}\right)=0 \\
& \frac{1}{\sqrt{V}} \partial_{y}\left[\frac{f_{12}-f_{3123}}{\sqrt{V}}\right]+\frac{1}{\sqrt{V}} \partial_{z}\left[\frac{f_{13}+f_{2123}}{\sqrt{V}}\right]-\frac{1}{\sqrt{V}} \partial_{x}\left[\frac{f_{22}+f_{33}}{\sqrt{V}}\right]+4 F_{2}\left(f_{12}-f_{3123}\right)-4 F_{3}\left(f_{13}+f_{2123}\right)=0
\end{aligned}
$$




$$
\begin{gathered}
\frac{1}{\sqrt{V}} \partial_{z}\left[\frac{f_{23}-f_{1123}}{\sqrt{V}}\right]+\frac{1}{\sqrt{V}} \partial_{x}\left[\frac{f_{21}+f_{3123}}{\sqrt{V}}\right] \\
-\frac{1}{\sqrt{V}} \partial_{y}\left[\frac{f_{33}+f_{11}}{\sqrt{V}}\right]+4 F_{3}\left(f_{23}-f_{1123}\right) \\
-4 F_{1}\left(f_{21}+f_{3123}\right)=0
\end{gathered}
$$

The three equations $\mathcal{F E}_{023}^{\gamma_{1} \gamma_{2} \gamma_{3}}, \mathcal{F} \mathcal{E}_{031}^{\gamma_{1} \gamma_{2} \gamma_{3}}, \mathcal{F} \mathcal{E}_{012}^{\gamma_{1} \gamma_{2} \gamma_{3}}$ are

$$
\begin{aligned}
& \frac{1}{\sqrt{V}} \partial_{z}\left[\frac{h_{31}-f_{2123}}{\sqrt{V}}\right]-\frac{1}{\sqrt{V}} \partial_{y}\left[\frac{h_{12}-f_{3123}}{\sqrt{V}}\right] \\
& \quad+F_{2}\left(h_{12}+3 f_{3123}-h_{0}+f_{33}\right) \\
& \quad-F_{3}\left(h_{31}+3 f_{2123}+h_{23}+f_{23}\right)+4 F_{1} h_{12}=0
\end{aligned}
$$

$$
\begin{aligned}
& \frac{1}{\sqrt{V}} \partial_{x}\left[\frac{h_{12}-f_{3123}}{\sqrt{V}}\right]-\frac{1}{\sqrt{V}} \partial_{z}\left[\frac{h_{23}-f_{1123}}{\sqrt{V}}\right] \\
& \quad+F_{3}\left(h_{23}+3 f_{1123}-h_{0}+f_{11}\right) \\
& \quad-F_{1}\left(h_{12}+3 f_{3123}+h_{31}+f_{31}\right)+4 F_{2} h_{23}=0
\end{aligned}
$$

$$
\begin{aligned}
& \frac{1}{\sqrt{V}} \partial_{y}\left[\frac{h_{23}-f_{1123}}{\sqrt{V}}\right]-\frac{1}{\sqrt{V}} \partial_{x}\left[\frac{h_{31}-f_{2123}}{\sqrt{V}}\right] \\
& \quad+F_{1}\left(h_{31}+3 f_{2123}-h_{0}+f_{22}\right) \\
& \quad-F_{2}\left(h_{23}+3 f_{1123}+h_{12}+f_{12}\right)+4 F_{3} h_{31}=0
\end{aligned}
$$

[1] S. Ferrara and P. van Nieuwenhuizen, Consistent Supergravity with Complex Spin 3/2 Gauge Fields, Phys. Rev. Lett. 37, 1669 (1976).

[2] S. D. Majumdar, A class of exact solutions of Einstein's field equations, Phys. Rev. 72, 390 (1947).

[3] A. Papapetrou, Einstein's theory of gravitation and flat space, Proc. R. Irish Acad. A 52, 11 (1948), https:// inspirehep.net/literature/44817.

[4] G. W. Gibbons and C. M. Hull, A Bogomolny bound for general relativity and solitons in $N=2$ supergravity, Phys. Lett. 109B, 190 (1982).

[5] C. M. Hull, The positivity of gravitational energy and global supersymmetry, Commun. Math. Phys. 90, 545 (1983).

[6] K.p. Tod, All metrics admitting supercovariantly constant spinors, Phys. Lett. 121B, 241 (1983).

[7] They have computed the full fermionic solution, not just up to the linearized order, which is what we restrict ourselves to in this paper.

[8] P. C. Aichelburg and R. Gueven, Supersymmetric Black Holes in $N=2$ Supergravity Theory, Phys. Rev. Lett. 51, 1613 (1983).

[9] P. C. Aichelburg and F. Embacher, The exact superpartners of $N=2$ supergravity solitons, Phys. Rev. D 34, 3006 (1986).

[10] P. C. Aichelburg and F. Embacher, Supergravity solitons. 1. General framework, Phys. Rev. D 37, 338 (1988).

[11] P. C. Aichelburg and F. Embacher, Supergravity solitons. 2. The free case, Phys. Rev. D 37, 911 (1988).

[12] P. C. Aichelburg and F. Embacher, Supergravity solitons. 3. The background problem, Phys. Rev. D 37, 1436 (1988).

[13] P. C. Aichelburg and F. Embacher, Supergravity solitons. 4. Effective soliton interaction, Phys. Rev. D 37, 2132 (1988).

[14] F. Embacher, Grassmann expansion of the classical $N=2$ supergravity field equations, Classical Quantum Gravity 2, 323 (1985).

[15] P. C. Aichelburg and H. K. Urbantke, Necessary and sufficient conditions for trivial solutions in supergravity, Gen. Relativ. Gravit. 13, 817 (1981).
[16] We work with the conventions:

$$
\begin{aligned}
& \gamma_{0}=\left(\begin{array}{ll}
0 & 1 \\
1 & 0
\end{array}\right), \quad \gamma_{1}=\left(\begin{array}{cc}
0 & \sigma_{1} \\
-\sigma_{1} & 0
\end{array}\right), \\
& \gamma_{2}=\left(\begin{array}{cc}
0 & \sigma_{2} \\
-\sigma_{2} & 0
\end{array}\right), \gamma_{3}=\left(\begin{array}{cc}
0 & \sigma_{3} \\
-\sigma_{3} & 0
\end{array}\right), \\
& \sigma_{a b}=\frac{1}{4}\left[\sigma_{a}, \sigma_{b}\right], \quad \gamma_{5}=i \gamma_{0} \gamma_{1} \gamma_{2} \gamma_{3},
\end{aligned}
$$

[17] R. Brooks, R. Kallosh, and T. Ortin, Fermion zero modes and black hole hypermultiplet with rigid supersymmetry, Phys. Rev. D 52, 5797 (1995).

[18] B. Bates and F. Denef, Exact solutions for supersymmetric stationary black hole composites, J. High Energy Phys. 11 (2011) 127.

[19] S. Banerjee, A. Sen, and Y. K. Srivastava, Generalities of quarter BPS dyon partition function and dyons of torsion two, J. High Energy Phys. 05 (2008) 101.

[20] A. Dabholkar, J. Gomes, and S. Murthy, Counting all dyons in $N=4$ string theory, J. High Energy Phys. 05 (2011) 059.

[21] A. Dabholkar, J. Gomes, S. Murthy, and A. Sen, Supersymmetric index from black hole entropy, J. High Energy Phys. 04 (2011) 034.

[22] G. Bossard and S. Lust, Microstate geometries at a generic point in moduli space, Gen. Relativ. Gravit. 51, 112 (2019).

[23] P. Dubey and C. N. Gowdigere (to be published).

[24] This term is borrowed from Ref. [17].

[25] S. Weinberg, The Quantum Theory of Fields (Cambridge University Press, Cambridge, 1995), https://doi.org/ 10.1017/CBO9781139644167.

[26] P. Van Nieuwenhuizen, Supergravity, Phys. Rep. 68, 189 (1981).

[27] D. Freedman and A. Van Proeyen, Supergravity (Cambridge University Press, Cambridge, 2012), https://doi.org/ 10.1017/CBO9781139026833.

[28] L. Baulieu, Unimodular gauge in perturbative gravity and supergravity, Phys. Lett. B 808, 135591 (2020). 
[29] A. Einstein, Die Grundlage der Allgemeinen Relativitatstheorie, Ann. Phys. (N.Y.) 354, 769 (1916).

[30] C. Barbarosie, Representation of divergence-free vector fields, Q. Appl. Math. 69, 309 (2011).

[31] We thank Prof. Ashoke Sen for the contents of this subsection.

[32] R. Kallosh and A. D. Linde, Black hole superpartners and fixed scalars, Phys. Rev. D 56, 3509 (1997).

[33] J. P. Gauntlett, J. B. Gutowski, C. M. Hull, S. Pakis, and H. S. Reall, All supersymmetric solutions of minimal supergravity in five- dimensions, Classical Quantum Gravity 20, 4587 (2003).

[34] J. C. Breckenridge, R. C. Myers, A. W. Peet, and C. Vafa, D-branes and spinning black holes, Phys. Lett. B 391, 93 (1997).

[35] H. Elvang, R. Emparan, D. Mateos, and H. S. Reall, A Supersymmetric Black Ring, Phys. Rev. Lett. 93, 211302 (2004).
[36] S. Chandrasekhar, The Mathematical Theory of Black Holes (Clarendon Press, 1998).

[37] E. Berti, V. Cardoso, and A. O. Starinets, Quasinormal modes of black holes and black branes, Classical Quantum Gravity 26, 163001 (2009).

[38] P. C. Aichelburg and R. Gueven, Can charged black holes have a superhair? Phys. Rev. D 24, 2066 (1981).

[39] G. F. Torres Del Castillo, Spin $3 / 2$ perturbations of algebraically special solutions of the Enstein-Maxwell equations, J. Math. Phys. (N.Y.) 30, 2114 (1989).

[40] H. T. Cho, Asymptotic quasinormal frequencies of different spin fields in spherically symmetric black holes, Phys. Rev. D 73, 024019 (2006).

[41] C. H. Chen, H. T. Cho, A. S. Cornell, G. Harmsen, and X. Ngcobo, Quasinormal modes and absorption probabilities of spin- $3 / 2$ fields in D-dimensional ReissnerNordström black hole spacetimes, Phys. Rev. D 97, 024038 (2018). 